\title{
CARACTERIZAÇÃO DE IDOSOS SINTOMÁTICOS OU PORTADORES DE AFECÇÕES NEUROPSIQUIÁTRICAS SEGUNDO CUIDADORES FAMILIARES
}

\author{
Hemília Gabrielly de Oliveira Cartaxo¹, Mércia Maria Paiva Gaudêncio², Gersiane da Silva Lacerda ${ }^{3}$
}

\begin{abstract}
RESUMO: Trata-se de um estudo descritivo, quantitativo, que caracterizou idosos dependentes com sintomas ou portadores de afecções neuropsiquátricas, segundo seus cuidadores familiares. Teve por sujeitos cuidadores familiares de idosos dependentes adscritos a uma Unidade Básica de Saúde da Família. Os dados foram coletados por meio de formulário de informações e analisados por estatística descritiva. Os idosos eram em sua maioria do sexo masculino, casados, com idade média de 72 anos; e majoritariamente aposentados que trabalharam na construção civil. Apresentam, segundo os cuidadores, sintomas de uma ou mais afecção neuropsiquiátrica, com destaque para a cefaléia; a afecção mais frequente foi o acidente vascular encefálico. Considerando os índices de dependência gerados pelo acidente vascular encefálico evidencia-se a necessidade de conhecer o perfil dos idosos afetados por esta condição e por sintomas neuropsiquiátricos, objetivando orientar a atuação dos profissionais da atenção básica.
\end{abstract}

PALAVRAS-CHAVE: Envelhecimento; Idoso; Cuidadores.

\section{CHARACTERIZATION OF ELDERLY PERSONS WHO ARE SYMPTOMATIC OR ACTUAL CARRIERS OF NEUROPSYCHIATRIC CONDITIONS ACCORDING TO FAMILY CARERS}

ABSTRACT: A descriptive, quantitative study which characterizes dependent elderly persons who have symptoms of or are actually suffering from neuropsychiatric conditions according to their families. As its study subjects the research had family carers of dependent elderly persons, on the books of a Basic Family Health Unit. The data were collected via an information form and analyzed by descriptive statistics. The majority of the elderly persons were masculine, married, and with an average age of 72; they were mainly retired or working in civil construction. According to their carers they presented one or more neuropsychiatric conditions with emphasis on severe headaches; the most common condition was the cerebrovascular accident. Considering the high levels of dependency caused by CVAs, it is evident that it is necessary to be familiar with the profile of elderly persons affected by this condition and by neuropsychiatric symptoms, in order to direct the actions of health professionals in basic care.

KEYWORDS: Aging; Elderly; Carers.

\section{CARACTERIZACIÓN DE ANCIANOS SINTOMÁTICOS O PORTADORES DE AFECCIONES NEUROPSIQUIÁTRICAS SEGÚN CUIDADORES FAMILIARES}

\begin{abstract}
RESUMEN: Es un estudio descriptivo, cuantitativo, que ha caracterizado ancianos dependientes con síntomas o portadores de afecciones neuropsiquátricas, según sus cuidadores familiares. Tuvo por sujetos cuidadores familiares de ancianos dependientes de una Unidad Básica de Salud de la Familia. Los datos fueron recogidos por medio de formulario de informaciones y analizados por estadística descriptiva. Los ancianos eran, en mayoría, del sexo masculino, casados, con edad media de 72 años; y en la mayor parte jubilados que trabajaron en la construcción civil. Presentan, de acuerdo a los cuidadores, síntomas de una o más afección neuropsiquiátrica, con destaque para a cefalea; la afección más frecuente fue el accidente vascular encefálico. Considerando los índices de dependencia generados por el accidente vascular encefálico, se evidencía la necesidad de conocer el perfil de los ancianos afetados por esta condición y por síntomas neuropsiquiátricos, objetivando orientar la actuación de los profesionales de la atención básica.
\end{abstract}

PALABRASCLAVE: Envelhecimento; Anciano; Cuidadores.

\footnotetext{
*Extraído da Monografia de Conclusão do Curso de Graduação em Enfermagem na Universidade Estadual da Paraíba - UEPB.

${ }^{1}$ Enfermeira. Especialista em Saúde Mental. Mestranda em Enfermagem pela UEPB.

${ }^{2}$ Enfermeira. Psicóloga. Mestre em Saúde Coletiva. Professora do Departamento e Mestrado em Enfermagem - UEPB.

${ }^{3}$ Enfermeira.
}

Autor correspondente:

Hemília Gabrielly de Oliveira Cartaxo

Universidade Estadual da Paraíba

Endereço: R. Cristino Collaço, 268 - 58103-313 - Campina Grande-PB-Brasil

E-mail: hemiliagoc@hotmail.com

Recebido: $29 / 07 / 10$

Aprovado: $12 / 05 / 11$ 


\section{INTRODUÇÃO}

A expectativa de vida no Brasil vem aumentando rápida e progressivamente; esta acelerada mudança no perfil epidemiológico gera desafios médicos e socioeconômicos ${ }^{(1)}$. Um destes desafios é posto quando se verifica que pessoas idosas tendem a usar com maior frequência os serviços de saúde. As doenças que prevalecem entre idosos são crônicas e múltiplas, o que exige, habitualmente, acompanhamento médico e de uma equipe multidisciplinar, visto que tais patologias podem gerar incapacidades ou requisitam cuidados em longo prazo. As doenças que mais acometem idosos e que são apontadas como as três principais causas de mortes são câncer, doença cardíaca e acidente vascular encefálico $(\mathrm{AVE})^{(2)}$.

Considerando as queixas de saúde apresentadas pelos idosos, é observado que as de natureza neuropsiquiátricas destacam-se entre as demais. Segundo estimativas, no Brasil, 11,5\% das doenças são de ordem neuropsiquiátrica, sendo os transtornos mentais aqueles que comprometem mais severamente a vida de relações ${ }^{(3)}$. As queixas de natureza psíquica, associadas às afecções neuropsiquiátricas, levaram o Ministério da Saúde a afirmar que os problemas de saúde mental têm aumentado de modo dramático. Esta é uma das razões pelas quais a ansiedade, os estados confusionais agudos, o alcoolismo, a melancolia e a solidão transitam na clínica, ora como sintomas, ora como patologias neuropsiquiátricas ${ }^{(4)}$.

Torna-se importante, diante destas observações, difundir ideias e propor ações de caráter preventivo, com o objetivo de educar a população acerca das relações entre apresentar sintomas e desenvolver patologias neuropsiquiátricas. A intervenção precoce em qualquer processo patológico - em especial no tocante às doenças neuropsiquiátricas - tende a beneficiar tanto o indivíduo quanto o grupo familiar.

Quando um membro de uma família assume os cuidados de um idoso dependente, passa a ser denominado cuidador familiar, para desempenhar adequadamente esta função necessita desenvolver uma série de habilidades, dentre as quais destaca-se: a comunicação, as técnicas para a realização da higiene, dos cuidados com a pele, para alimentá-lo adequadamente, realizar atividades físicas, administrar corretamente os medicamentos e saber lidar com episódios de desconforto e dor ${ }^{(5)}$.

$\mathrm{Na}$ mesma proporção que se tem ampliado a expectativa de vida de brasileiros tem aumentado o número de familiares que passam à condição de cui- dadores de idosos dependentes. Na maioria dos casos, o familiar cuidador não recebe apoio nem orientações profissionais adequadas para desenvolver os cuidados. O limitado amparo social, associado à desinformação e aos parcos recursos econômicos, dificulta a assistência domiciliar a idosos dependentes. Assim, o somatório destas condições, aliado ao prognóstico das patologias neuropsiquiátricas, conduz os familiares a vivenciar o cuidar como uma sobrecarga. Estas circunstâncias, portanto, fragilizam os cuidadores familiares e interferem em sua qualidade de vida e, consequentemente, na dos idosos que recebem cuidados ${ }^{(6)}$.

Refletindo acerca dos fatores e condições acima expostos e, simultaneamente, sobre a assistência prestada na Atenção Básica, tanto aos idosos quanto aos familiares cuidadores, recorremos aos últimos, enquanto informantes privilegiados, para coletar dados socioeconômicos dos idosos sintomáticos ou portadores de afecções neuropsiquiátricas. É nosso objetivo caracterizá-los e, assim, sistematizar informações que possam ser utilizadas tanto para a contextualização dos envolvidos nesta relação de cuidados, quanto no planejamento da assistência a ser prestada ao binômio constituído pelo idoso dependente e o cuidador familiar.

\section{METODOLOGIA}

Este é um estudo descritivo, exploratório e de campo, com abordagem quantitativa, por meio do qual caracterizamos idosos dependentes que apresentaram sintomas ou afecções neuropsiquiátricas. Para tanto, tomamos como sujeitos desta investigação cuidadores familiares de idosos dependentes adscritos a uma Unidade Básica de Saúde da Família (UBSF), localizada na Cidade de Campina Grande, Estado da Paraíba. A escolha dos familiares como sujeitos desta investigação deveu-se ao fato destes serem considerados informantes privilegiados em relação aos idosos, uma vez que cuidam cotidianamente destas pessoas e poderiam fornecer informações, caso os idosos apresentassem comprometimento cognitivo que inviabilizasse a coleta de dados.

Os critérios de inclusão no estudo foram: ser maior de idade; residir com o idoso; identificar-se como cuidador do idoso; e responsabilizar-se pelas tarefas cotidianas do cuidar há pelo menos seis meses. Obedecendo a estes critérios, reunimos uma amostra composta por 13 cuidadores familiares, responsáveis pelos cuidados domiciliares prestados a 14 idosos.

Os dados foram coletados entre janeiro e março de 2009 por ocasiões de visitas domiciliares previa- 
mente agendadas na UBSF e por meio de formulário de informações socioeconômicas subdividido em duas partes. Na primeira parte do instrumento foram coletadas variáveis socioeconômicas dos cuidadores familiares e dos idosos, e na segunda parte foram coletadas informações acerca de sintomas e de afecções neuropsiquiátricas que afetam os idosos. Os dados foram organizados e submetidos a procedimentos de estatística descritiva.

A pesquisa obedeceu às exigências da Resolução 196/96 do Conselho Nacional de Saúde, que trata das Diretrizes e Normas Regulamentadoras de Pesquisa envolvendo seres humanos ${ }^{(7)}$. Antecedendo a coleta de dados, o projeto foi aprovado pelo Comitê de Ética em Pesquisa da Universidade Estadual da Paraíba, sob número 0552.0.133.000-08.

\section{RESULTADOS}

Inicialmente, apresentamos as variáveis socioeconomicas que permitiram caracterizar os idosos e os cuidadores familiares. Assim procedemos para ter acesso ao contexto no qual estes indivíduos estão inseridos e, a partir daí, apreender e dimensionar a amplitude dos problemas que os afligem.

Os familiares nos forneceram informações acerca da idade dos idosos, a qual variou entre 60 e 90 anos, sendo a média de idade de 72,6 anos. Em seguida, na tabela 1, apresentamos a distribuição dos idosos por estado conjugal e sexo.

Constatamos a predominância $(78,6 \%)$ de idosos do sexo masculino, quando comparada à presença de mulheres $(21,4 \%)$. Considerando o número total

Tabela 1 - Distribuição dos idosos por estado conjugal e sexo, segundo informações dos cuidadores familiares. Campina Grande, 2009

\begin{tabular}{lcccccc}
\hline Estado conjugal & \multicolumn{2}{c}{ Feminino } & \multicolumn{2}{c}{ Masculino } & \multicolumn{2}{c}{ Total } \\
& $\mathbf{N}$ & $\mathbf{\%}$ & $\mathbf{N}$ & $\mathbf{\%}$ & $\mathbf{N}$ & $\%$ \\
\hline Casado & 1 & 7,1 & 6 & 42,9 & 07 & 50 \\
Viúvo & 2 & 14,3 & 2 & 14,3 & 04 & 28,6 \\
Separado & - & - & 2 & 14,3 & 02 & 14,3 \\
Solteiro & - & - & 1 & 7,1 & 01 & 7,1 \\
\hline Total & 3 & 21,4 & 11 & 78,6 & 14 & 100,0 \\
\hline
\end{tabular}

de idosos (14), evidencia-se que metade (50\%) é casada e que este estado conjugal predomina entre os homens; entre as mulheres prevalece o estado conjugal de viúvas.

Posteriormente, buscando conhecer um pouco do passado dos idosos, no tocante às formas como garantiam a sua sobrevivência e a dos familiares, para isso indagamos os cuidadores sobre a profissão/ocupação destas pessoas quando estavam inseridos no mercado de trabalho, dados apresentados na tabela 2 .
Constatamos que o número total de profissões/ocupações identificadas é superior ao número de idosos, uma vez que um dos familiares afirmou que o idoso sobre seus cuidados exerceu mais de uma profissão/ ocupação. Ao cruzarmos as informações referentes à profissão/ocupação com o gênero constatamos que o trabalho doméstico foi exercido exclusivamente por mulheres.

Em íntima associação com a profissão/ocupação, investigamos a distribuição dos idosos quanto à renda

Tabela 2 - Distribuição dos idosos quanto à profissão/ocupação que realizavam antes de se tornarem dependentes, segundo informações dos cuidadores familiares. Campina Grande, 2009

\begin{tabular}{lccc}
\hline Categoria do trabalho & Profissão/Ocupação & N & \% \\
\hline Construção Civil & $\begin{array}{c}\text { Pintor, Pedreiro, } \\
\text { Marceneiro, Carpinteiro }\end{array}$ & 08 & 53,4 \\
\hline Trabalho Doméstico & $\begin{array}{c}\text { Dona-de-casa, } \\
\text { Empregada doméstica }\end{array}$ & 02 & 13,3 \\
\hline Agricultura & Agricultor & 02 & 13,3 \\
\hline Comércio & Vendedor, Marchante & 02 & 13,3 \\
\hline Segurança & Vigilante & 01 & 6,7 \\
\hline Total & - & 15 & 100 \\
\hline
\end{tabular}


mensal, quando verificamos que são na totalidade aposentados. Salientamos que a aposentadoria dos idosos compõe a renda familiar, sendo esta, por vezes, a única fonte de sustento de toda a família.

Em seguida, na tabela 3, estão expostos os principais sintomas e afecções neuropsiquiátricas detectados nos idosos, segundo a percepção dos cuidadores familiares. Observamos que o AVE foi a patologia mais referida pelos cuidadores familiares $(44,6 \%)$. No tocante aos sintomas neuropsiquiátricos, a cefaleia alcançou maior percentagem, atingindo 16,9\%.

Tabela 3 - Afecções neuropsiquiátricas e sintomas dos idosos dependentes. Campina Grande, 2009

\begin{tabular}{lccc}
\hline & & N & \% \\
\hline \multirow{2}{*}{ Afecções } & Acidente Vascular & 08 & 44,6 \\
Neuropsiquiá- & Encefálico & & \\
tricas & Alcoolismo & 01 & 5,5 \\
& Mal de Parkinson & 01 & 5,5 \\
& Depressão & 01 & 5,5 \\
\hline Subtotal & - & 12 & 66,6 \\
\hline \multirow{3}{*}{ Sintomas Neu- } & Cefaleia & 03 & 16,9 \\
ropsiquátricos & Dormência nas extre- & 01 & 5,5 \\
& midades inferiores & 01 & 5,5 \\
& Crises convulsivas & 01 & 5,5 \\
\hline Subtotal & - & 06 & 33,4 \\
\hline TOTAL & - & 18 & 100 \\
\hline
\end{tabular}

Encerramos a apresentação dos resultados caracterizando os cuidadores familiares, uma vez que são agentes dos cuidados prestados no domicílio aos idosos e, também, informantes privilegiados para a questão que guia este estudo. A média de idade das cuidadoras era de 44 anos, no entanto, destacamos que a maioria $(38,4 \%)$ estava na faixa etária compreendida entre 20 30 anos, o que nos permite caracterizá-las como jovens cuidadoras. No tocante ao estado conjugal, $53,8 \%$ são solteiras e $76,9 \%$ filhas dos idosos; $92,3 \%$ possuem renda familiar mensal equivalente a 2 salários mínimos nacionais e destas, 53,8\% possíam como nível de escolaridade máximo o Ensino Fundamental Incompleto.

\section{DISCUSSÃO}

Embora conscientes que nossos resultados não são passíveis de generalizações, é curioso verificar que no tocante à variável sexo, predomina o sexo masculino
$(78,6 \%)$, sendo este um resultado controverso em relação às pesquisas realizadas no Brasil, nas quais a maioria é mulher. Em relação às pessoas dependentes, existe uma diferença significativa em relação ao gênero em quase todas as idades, especialmente nas mais avançadas, devido, provavelmente, à sobremortalidade masculina ${ }^{(8)}$.

Para discutir o estado conjugal dos idosos, julgamos pertinente considerar que, no Brasil, mais de 3/4 dos homens vivem em companhia de cônjuge, enquanto $2 / 3$ das mulheres são viúvas, solteiras ou separadas ${ }^{(9)}$. No presente estudo estas informações foram confirmadas, visto que os idosos do sexo masculino, em sua maioria, eram casados.

Considerando a média de idade dos idosos (72,6 anos), verificamos que pessoas nesta faixa etária ultrapassaram a expectativa de vida do brasileiro ao nascer que é de 72,3 anos $^{(1)}$. Para compreendermos adequadamente o sentido desta informação é interessante lembrarmos que não há um padrão universalmente aceito para definir o início do processo de envelhecimento. Por esta razão, consultamos uma das classificações do envelhecimento que subdivide-se em cinco categorias $^{(10)}$ : mediescência na fase I ( 30 a 49 anos de idade), pré-senescência ( 40 ou 45 a 59 ou 65 anos de idade), mediescência na fase II ( 50 a 65 ou 70 anos de idade), senescência ou terceira idade ( 60 ou 65 ou 70 a 75 ou 80 anos de idade) e, segunda velhice ou quarta idade (inicia-se aos 75 ou 80 anos).Partindo desta classificação, e considerando que na amostra estudada há idosos que atingiam a idade de 90 anos, podemos dizer que estes idosos se encontravam na segunda velhice ou quarta idade, sendo a fragilidade uma condição esperada nesta faixa etária. Assim, buscando esclarecimento acerca desta condição, considerou-se como idoso fragilizado aquele inserido na quarta idade, podendo ou não ter ultrapassado os 75 anos de idade e cuja fragilidade é caracterizada pela ocorrência de enfermidades e vulnerabilidade aos riscos que progressivamente o tornam incapaz de realizar atividades cotidianas ${ }^{(11)}$.

Ao investigar a profissão/ocupação dos idosos, verificamos que a maioria dos homens trabalhou na construção civil e que existem associações entre as atividades desenvolvidas neste setor e as camadas mais pobres da população. É ressaltado que este setor lidera as taxas de acidentes de trabalho fatais e não fatais e, também, de anos de vida perdidos ${ }^{(12)}$. Estas informações, principalmente no tocante ao gênero e às condições econômicas, se aplicam aos sujeitos investigados. 
Por outro lado, o trabalho doméstico, como apresentado nos resultados, foi exercido exclusivamente por mulheres. Esta ocupação tem sido historicamente desvalorizada por se tratar de atividades que não requerem qualificação específica para seu exercício. Embora as atividades tidas como exclusivamente femininas estejam passando por uma modificação no plano das representações sociais, observamos que culturalmente as "atividades domésticas" ainda são dotadas de um caráter de gratuidade, não-produtividade e invisibilidade social. Inferimos que, embora tenhamos na atualidade novas proposituras para o trabalho doméstico, as idosas que pesquisamos não usufruíram dos benefícios resultantes das novas concepções do trabalho doméstico.

A literatura corrobora o fato de a totalidade ser mulher cuidadora; cabe às mulheres, no contexto familiar, realizar as atividades da vida diária dirigidas às pessoas que necessitam de cuidados ${ }^{(11)}$. Notamos, ainda, que elas são jovens e, em sua maioria solteiras e filhas dos idosos por elas cuidadas, reforçando o contexto do papel de familiar cuidadora.

Ao discutir as afecções neuropsiquiátricas, devemos lembrar que o AVE é uma das doenças neurológicas prevalentes e que, segundo os cuidadores familiares, esta foi a patologia que mais acometeu os idosos. Neste sentido, é interessante lembrar que, nos países desenvolvidos esta é a terceira causa de morte neste extrato etário da população. Por esta razão, colocamos em destaque as seguintes estatísticas: aproximadamente $20 \%$ dos idosos que sofreram AVE vão a óbito dentro de um mês; cerca de $50 \%$ dos sobreviventes apresentam incapacidades permanentes e significativas, demandando assistência e supervisão para execução de atividades básicas; os outros 30\% apresentam déficit neurológico, mas são capazes de viver de forma independente ${ }^{(13)}$.

Uma cuidadora, ao fazer referência a problemas mentais, não especificou um transtorno em particular, nem um sintoma específico. Inferimos que esta poderia ser uma referência associada aos idosos que apresentam quadros demenciais, uma vez que estes se caracterizam por déficit de memória, dificuldades de executar tarefas domésticas, problema com o vocabulário, desorientação, perda da capacidade de julgar situações, alterações de humor e de comportamento. O número de sintomas associados à gravidade dos mesmos tende a tornar o idoso dependente de cuidados, visto que, com o progresso destes sintomas, o indivíduo perde, paulatinamente, a autonomia ${ }^{(14)}$.

Ressaltamos que, do ponto de vista leigo, os sin- tomas neuropsiquiátricos são, com frequencia, interpretados como parte do processo natural de envelhecimento. Inferimos que o cuidador leigo experimenta dificuldades para diferenciar o que é normal ou patológico no envelhecimento e que esta indefinição pode induzir a erros no cuidado da pessoa idosa e retardar diagnósticos.

A mesma compreensão pode ser aplicada às denominadas patologias urbanas, tais como a depressão e o alcoolismo. O uso abusivo do álcool, em associação com as mudanças fisiológicas do envelhecimento, tende a causar uma diminuição na capacidade do organismo em tolerar o álcool ${ }^{(15)}$. Na população estudada, registramos um baixo percentual $(5,5 \%)$ de referências ao alcoolismo e à depressão.

As cuidadoras fizeram referência à Doença de Parkinson, razão pela qual lembramos que a literatura aponta que essa doença atinge frequentemente pessoas com idade superior a 50 anos. Suas características clínicas são: tremor de repouso de baixa amplitude, assimétrico, que acomete só um membro ou os membros de um dos lados; movimento rítmico dos dedos das mãos; bradicinesia; rigidez; síndrome das pernas inquietas, entre outros ${ }^{(16)}$. Evidenciam-se nesta descrição os desconfortos vivenciados pelos portadores da Doença de Parkinson. Também é possível inferir que o familiar que cuida de um idoso portador desta patologia experimenta sentimentos de impotência.

Finalizando a categorização das afecções neuropsiquiátricas apontadas pelos cuidadores familiares dos idosos, foram feitas referências à depressão. Esta é considerada a doença psiquiátrica mais comum entre idosos, embora, com frequência, permaneça sem diagnóstico e tratamento. A patologia afeta tão gravemente a qualidade de vida dos portadores que se deve considerar a instalação de tendências suicidas. Também há que se considerar que a insatisfação, o isolamento e, consequentemente, a privação das relações interpessoais, podem encurtar a expectativa de vida, seja pela possibilidade de suicídio ou pela instalação de doenças somáticas relacionadas à depressão ${ }^{(17)}$.

No que se refere aos sintomas neuropsiquiátricos que acometem os idosos, verificamos que a cefaleia ocupa o primeiro lugar entre as queixas dos idosos. Esta condição é considerada como um sintoma comum, independentemente da faixa etária e da condição física em que se encontra a pessoa com este sintoma. Entre os idosos investigados em outro estudo, a prevalência da cefaleia foi de $55 \%$ entre mulheres e de $45 \%$ entre homens ${ }^{(18)}$. Nossos dados, quando comparados aos 
anteriormente descritos, apresentam particularidades, uma vez que os cuidadores familiares relataram que dos 14 idosos investigados, apenas três - um do sexo feminino e dois do sexo masculino - se queixam desse sintoma.

A insônia é considerada o sintoma mais comumente referido por pessoas idosas, no entanto, segundo relato das cuidadoras, esta queixa ocupa, percentualmente, o segundo lugar dentre os sintomas aludidos pelos idosos. Segundo especialistas, as pessoas com quadros demenciais podem vir a apresentar distúrbios do ciclo do sono, gerando problemas familiares e desgaste do cuidador $^{(15)}$.

Ao se referir à dormência nas extremidades inferiores como queixa comum entre os idosos, as cuidadoras não reconhecem nesta manifestação um sintoma. A dormência nos pés ou parestesia, consiste em uma sensação subjetiva de desconforto. Esta sensação pode estar relacionada a uma lesão muscular, articular ou vascular, necessitando, por isto, de investigação acurada. São quatro os diagnósticos clínicos que devem ser pesquisados no idoso: neuropatia periférica, fibromialgia, síndrome miofascial e doença arterial periférica ${ }^{(19)}$.

As crises convulsivas aparecem nas palavras das cuidadoras como uma patologia e não como sintoma associado a outras enfermidades. Aparentemente existem poucos estudos acerca das particularidades das crises convulsivas neste grupo etário, no entanto, sabe-se que são produzidas por qualquer irritação no cérebro ${ }^{(20)}$.

Todos os idosos, em conformidade com as informações prestadas pelos cuidadores, apresentam algum grau de dependência (leve, moderada ou grave). Embora este problema não tenha sido adequadamente investigado, é possível inferir que as limitações apresentadas pelos idosos comprometem sua autonomia para alimentação e hidratação, higiene, locomoção e realização de algumas atividades domésticas consideradas pesadas, tais como lavar e passar roupas, varrer e mover objetos pesados.

\section{CONSIDERAÇÕES FINAIS}

Em conformidade com os resultados e discussões empreendidas ao longo deste trabalho, tivemos acesso à realidade que atinge tanto os idosos quanto seus cuidadores familiares. O conhecimento resultante da caracterização dos idosos e dos cuidadores familiares nos permite afirmar que é importante que estes recebam suporte - em especial dos profissionais de saúde da Atenção Básica - para desenvolver adequadamente os cuidados aos idosos dependentes.

A precariedade da condição socioeconômica, associada à baixa escolaridade e às limitações no atendimento médico e de enfermagem, colocam os idosos e cuidadores familiares em situação de risco físico e mental. Neste sentido, o planejamento de políticas públicas que considerem as particularidades socioeconômicas das populações e que guiem intervenções dos profissionais de saúde é essencial para a manutenção e promoção da qualidade de vida das pessoas envolvidas nas responsabilidades e tarefas do cuidar de idosos dependentes no domicílio.

Também vislumbramos que é urgente atender à demanda dos cuidadores familiares, uma vez que a tendência, para os próximos anos, é de aumento expressivo no número de idosos, e com isso a possibilidade de instalação de doenças crônicas e múltiplas, capazes de gerar dependência.

\section{REFERÊNCIAS}

1. Instituto Brasileiro de Geografia e Estatística. Tábuas Completas de Mortalidade 2007. [acesso em 28 jun 2010]. Disponível: http://migre.me/51LJK

2. Pessini L, Barchifontaine CP. Bioética e longevidade humana. São Paulo: Loyola; 2006.

3. Ministério da Saúde. Governo do Estado de Goiás. Secretaria do Estado da Saúde. Superintendência de Políticas de Atenção Integral à Saúde. Política Estadual de Saúde Mental. Goiânia; 2003 [acesso em fev 2011]. Disponível: http://www.mp.go.gov.br/ portalweb/hp/8/docs/politica_estadual_de_saude mental___goias.pdf

4. Sociedade Brasileira de Geriatria Preventiva. Problemas geriátricos. A geriatria preventiva ajuda a evitá-los! 2003 [acesso em 26 mai 2009]. Disponível: http://www. sbgp.com.br/noticias/detalhes.asp?id=23

5. Costa R. A terceira idade hoje: sob a ótica do serviço social. Canoas: Ulbra; 2007.

6. Nardi EFR, Oliveira MLF. Conhecendo o apoio social ao cuidador familiar do idoso dependente. Rev Gaúch Enferm. 2008;29(1):47-53.

7. Ministério da Saúde (BR). Conselho Nacional de Saúde. Diretrizes e normas regulamentadoras sobre pesquisa envolvendo seres humanos. Resolução n. 196, de 10 de outubro de 1996. Brasília; 1996. 
8. Areosa SVC, Areosa AL. Envelhecimento e dependência: desafios a serem enfrentados. Revista Textos e Contextos. [Internet] 2008;7(1) [acesso em 11 maio 2010]. Disponível: http://migre.me/51LPP

9. Paschoal S, Franco RP, Salles RFN. Epidemiologia do envelhecimento. In: Papaléo Neto M. Tratado de gerontologia. $2^{\mathrm{a}}$ ed. São Paulo: Atheneu; 2007. p. 39-56.

10. Sene Costa EM. Gerontodrama: a velhice em cena. São Paulo: Ágora; 1998.

11. Gonçalves LHT, Silva YF, Pfeiffer S. O cuidado do idoso fragilizado e de seus cuidadores no contexto domiciliar. Cogitare Enferm. 1996;1(2):39-47.

12. Santana VS, Oliveira RP. Saúde e trabalho na construção civil em uma área urbana do Brasil. Cad Saúde Pública. [Internet] 2004;20(3) [acesso em 11 mai 2010]. Disponível: http://www.scielo.br/pdf/csp/ v20n3/17.pdf

13. Marques S, Rodrigues RAP, Kusumota L. O idoso após acidente vascular cerebral: alterações no relacionamento familiar. Rev Latino-Am Enfermagem. 2006;14(3):36471.

14. Ballone GJ, Moura EC. Demências. 2008 [acesso em 20 jan 2010]. Disponível: http://www.psiqweb.med.br/ site/?area $=\mathrm{NO} /$ LerNoticia\&idNoticia $=115$

15. Ham RJ. As queixas mais comuns dos idosos. In: Reichel W. Assistência ao idoso: aspectos clínicos do envelhecimento. Rio de Janeiro: Guanabara-Koogan; 2001. p. 68-72.

16. Finger G, Dietrich GV, Santos GT, Schmidt OF, Silva R, Silva S. Doença de Parkinson. In: Terra NL, Silva $\mathrm{R}$, Schmidt OF. Tópicos em geriatria II. Porto Alegre: EDIPUCRS; 2007. p. 113-5.

17. Oliveira DAAP, Gomes L, Oliveira RT. Prevalência de depressão em idosos que frequentam centros de convivência. Rev Saúde Pública. 2006;40(4):734-6.

18. Knoefel JE, Klein RB. Problemas neurológicos no idoso. In: Reichel W. Assistência ao idoso: aspectos clínicos do envelhecimento. Rio de Janeiro: GuanabaraKoogan; 2001. p. 200.

19. Berleze MCMC. Parestesias no idoso. In: Terra NL. Entendendo as queixas do idoso. Porto Alegre: EDIPUCRS; 2003. p. 95-6.

20. Xavier SM, Rocha MRS, Nakamura EK. Crise convulsiva e o Serviço de Atendimento Móvel de Urgência - SAMU. 2005 [acesso em 29 dez 2009]. Disponível: http://migre.me/51LTe 